\title{
Prognostic nutritional index predicts clinical outcomes in patients with cerebral venous sinus thrombosis
}

\author{
Jiawei Zhao ${ }^{\dagger}$, Kai Liư ${ }^{\dagger}$, Shen Li, Yuan Gao, Lu Zhao, Hongbing Liu, Hui Fang, Jun Wu, Shilei Sun, Yusheng Li,
} Bo Song ${ }^{*}$ and Yuming $\mathrm{Xu}^{*}$

\begin{abstract}
Background: Lower prognostic nutritional index (PNI) is related to the poor prognosis of cardiovascular diseases. However, little is known about PNI and its relationship with the prognosis of cerebral venous sinus thrombosis (CVST).

Methods: CVST patients were retrospectively identified from January 2013 till June 2019. Patients in the acute / subacute phase were selected as subjects. Poor prognosis was defined as a modified Rankin Scale (mRS) of 3-6. Multivariate logistic regression analysis was used to confirm if lower PNI was associated with a poor prognosis.

Results: A total of 297 subjects with follow-up data were enrolled. Thirty-three (11.1\%) had a poor outcome. Multivariate logistic regression analysis suggested that PNI was an important predictive factor of poor outcome in acute/ subacute CVST (odds ratio, $0.903 ; 95 \% \mathrm{Cl}, 0.833-0.978 ; P=0.012$ ). The optimal cut-off value for predicting the poor prognosis of PNI was 44.2. Kaplan-Meier analysis and log-rank test suggested that the lower the PNI value, the higher the mortality rate $(P<0.001)$. In addition, the nomogram that was set up showed that lower PNI was an index of poor prognosis. The c-index for acute/subacute patients with CVST was 0.872 .
\end{abstract}

Conclusion: Lower PNI is correlated with a higher risk of adverse clinical outcomes in patients with acute/subacute CVST.

Keywords: Prognostic nutritional index, Cerebral venous sinus thrombosis, Acute/subacute, Prognosis, Risk factors

\section{Background}

Cerebral venous sinus thrombosis (CVST) is a special type of cerebrovascular disease, which is characterized by cerebral venous reflux obstruction and intracranial hypertension caused by the disturbance of cerebrospinal fluid absorption. It is generally believed that the annual incidence of CVST is $2-5$ cases / million, accounting for $0.5-1 \%$ of all strokes [1-6]. A recent report suggested a much higher incidence (13 cases / million every years)

*Correspondence: fccsongb@zzu.edu.cn; xuyuming@zzu.edu.cn † Jiawei Zhao and Kai Liu contributed equally to this work.

Department of Neurology, the First Affiliated Hospital of Zhengzhou University, No.1 Eastern Jianshe Road, Zhengzhou 450052, Henan, China
[7]. The inducing risk factors, clinical characteristics, and neuroimaging features of CVST are extremely diverse, which cause challenges to the prognosis [8]. Therefore, it is clinically significant to carry out early risk stratification at the time of onset.

Malnutrition has been proven to be an independent prognostic index of incidence and mortality in patients with various cancers, myocardial infarction (MI) as well as acute ischemic stroke (AIS) [9-11]. The PNI is a novel and comprehensive nutritional-inflammatory score that reflects the immunological nutritional status based on serum albumin concentration and lymphocyte count. Several studies have found that PNI is related to poor prognosis and increased mortality in patients with 
malignant diseases, MI and AIS [12-14]. Nevertheless, this correlation between PNI and prognosis in CVST patients has not been studied yet. Acute/subacute CVST is characterized by a sudden onset and rapid progression, which is of great significance to study [15]. Therefore, the aim of this study was to assess the outcome and predictive role of PNI in patients with acute/subacute CVST.

\section{Material and methods}

\section{Patient selection}

A follow-up study was conducted from January 2013 till June 2019 at the Henan CVST Registry in the First Affiliated Hospital of Zhengzhou University (Henan, China). The diagnosis of CVST complied with international standards $[4,5]$, based on clinical symptoms, patient history, and neuroimaging results (computed tomographic (CT), magnetic resonance venography (MRV) or digital subtraction angiography (DSA)). The acute period defined as the time from onset to hospital admission was less than 7 days. Whereas subacute period is defined as the time from onset to hospital admission was less than 30 days [16, 17]. The exclusion criteria as follows: (1) patients without complete clinical data; (2) time from onset to admission over 30 days [15]; (3) patients less than 18 years old; (4) patients with severe hepatic or renal diseases; (5) patients lost to follow-up. The registry study was approved by the Ethics Committee of the First Affiliated Hospital of Zhengzhou University.

\section{Data collection}

Demographic data and information such as age, gender, risk factors, clinical manifestations, laboratorial parameters and imageological examination were gathered and analyzed. Albumin concentration (ALB) and the absolute lymphocyte count (ALC) were assessed $24 \mathrm{~h}$ after admission.

\section{Definition of PNI}

Similar to previous studies, PNI was calculated using 5 * lymphocyte count $\left(10^{9} / \mathrm{L}\right)+$ albumin concentration (g/L) [18].

\section{Outcome assessment}

The outcome was assessed by modified Rankin Scale (mRS) during routine follow-up, which was performed over the phone after 3 months of discharge. The adverse prognosis was defined as mRS of 3-6. Telephone interviewers were unaware of baseline data.

\section{Statistical analysis}

Statistical analysis was performed using SPSS 22.0. Continuous data were represented as mean \pm SD, which were analyzed by independent Student's t-test or MannWhitney U test, whichever was appropriate. Categorical variables were presented as numbers and percentages, which were compared by the $\chi^{2}$ test or Fisher exact test. Multivariate logistic regression analysis was used to analyze the association between PNI and clinical outcomes. The receiver operating characteristic (ROC) analysis was carried out to evaluate the ability of PNI in predicting clinical prognosis. The patients were dichotomized with the cut-off PNI value. The mortality rate in high and low PNI value groups was compared with the help of the log-rank test and plotted with the use of the Kaplan-Meier method. On this basis, a nomogram of independent predictive factors was established by using RMS software. The results were significant for Twotailed $P<0.05$.

Table 1 Baseline characteristics according to the clinical functional outcome

\begin{tabular}{|c|c|c|c|}
\hline & $\begin{array}{l}\text { Good Outcome } \\
(N=264)\end{array}$ & $\begin{array}{l}\text { Poor } \\
\text { Outcome } \\
(N=33)\end{array}$ & $P$ \\
\hline \multicolumn{4}{|l|}{ Demographics } \\
\hline Age, $y$, mean $\pm S D$ & $34.2 \pm 11.6$ & $44.4 \pm 16.3$ & $<0.001$ \\
\hline Female, n (\%) & 155(58.7) & 23(69.7) & 0.225 \\
\hline \multicolumn{4}{|l|}{ Possible Risk factors, n (\%) } \\
\hline Infections & $66(25.9)$ & $8(24.2)$ & 0.839 \\
\hline Pregnancy/postpartum & $66(25.0)$ & $7(21.2)$ & 0.634 \\
\hline \multicolumn{4}{|l|}{ Clinical symptoms, n (\%) } \\
\hline Intracranial hypertension & $175(66.3)$ & $22(66.7)$ & 0.965 \\
\hline Seizure & $74(28.0)$ & 12(36.4) & 0.320 \\
\hline Coma & $67(25.4)$ & $23(69.7)$ & $<0.001$ \\
\hline Mental status disturbance & $13(4.9)$ & $4(12.1)$ & 0.200 \\
\hline Focal neurological deficits ${ }^{\mathrm{a}}$ & $86(32.6)$ & 18(54.5) & 0.013 \\
\hline \multicolumn{4}{|l|}{ Involved sinuses, n (\%) } \\
\hline Transverse sinuses & $99(37.5)$ & 12(36.4) & 0.899 \\
\hline Sigmoid sinuses & 87(33.0) & $9(27.3)$ & 0.511 \\
\hline Superior sagittal sinus & $106(40.2)$ & 13(39.4) & 0.933 \\
\hline Straight sinus & $16(6.1)$ & $6(18.2)$ & 0.012 \\
\hline Inferior sagittal sinus & $18(6.8)$ & $3(9.1)$ & 0.904 \\
\hline Deep CVT & $9(3.4)$ & $2(6.1)$ & 0.786 \\
\hline \multicolumn{4}{|l|}{ Parenchymal lesion, n (\%) } \\
\hline Ischemic Infarction & $40(15.2)$ & $8(24.2)$ & 0.181 \\
\hline Intracerebral hemorrhage & $54(20.5)$ & $15(45.5)$ & 0.001 \\
\hline \multicolumn{4}{|l|}{ Laboratory Examinations } \\
\hline White Blood Cell, 109/L & $9.6 \pm 5.0$ & $10.9 \pm 5.5$ & 0.165 \\
\hline Lymphocyte count, $\times 10^{9} / \mathrm{L}$ & $1.6 \pm 0.8$ & $1.1 \pm 0.5$ & $<0.001$ \\
\hline Serum albumin, g/dL & $40.5 \pm 5.0$ & $37.4 \pm 5.0$ & 0.001 \\
\hline $\mathrm{PNI}$ & $49.3 \pm 6.3$ & $42.3 \pm 6.1$ & $<0.001$ \\
\hline \multicolumn{4}{|l|}{ Hospital treatment, n (\%) } \\
\hline Anticoagulation & 262(99.2) & $31(93.9)$ & 0.062 \\
\hline Endovascular Therapies & 125(47.3) & 19(57.6) & 0.268 \\
\hline
\end{tabular}

CVT cerebral venous thrombosis; PNI prognostic nutritional index ${ }^{a}$ Focal neurological deficits symptoms included hemiplegia and sensory changes 
Table 2 Multivariate logistic regression analysis of predictors for poor clinical outcome in CVST patients

\begin{tabular}{lllll}
\hline Model & Variable & OR & $95 \% \mathrm{Cl}$ & $\boldsymbol{P}$ \\
\hline Model 1 & Age & 1.083 & $1.045 \sim 1.122$ & $<0.001$ \\
(with ALC) & Gender & 2.102 & $0.787 \sim 5.615$ & 0.138 \\
& Coma & 6.455 & $2.497 \sim 16.687$ & $<0.001$ \\
& Focal neurological deficits* & 1.236 & $0.512 \sim 2.984$ & 0.638 \\
& Intracerebral hemorrhage & 3.765 & $1.464 \sim 9.683$ & 0.006 \\
& Straight sinus & 7.338 & $1.962 \sim 27.448$ & 0.003 \\
& ALC & 0.420 & $0.189 \sim 0.936$ & 0.034 \\
Model 2 & Age & 1.081 & $1.044 \sim 1.119$ & $<0.001$ \\
(with ALB) & Gender & 2.284 & $0.858 \sim 6.079$ & 0.098 \\
& Coma & 6.941 & $2.692 \sim 17.899$ & $<0.001$ \\
& Focal neurological deficits* & 1.273 & $0.527 \sim 3.079$ & 0.592 \\
& Intracerebral hemorrhage & 3.585 & $1.408 \sim 9.128$ & 0.007 \\
& Straight sinus & 9.006 & $2.272 \sim 35.695$ & 0.002 \\
& ALB & 0.923 & $0.840 \sim 1.015$ & 0.100 \\
Model 3 & Age & 1.080 & $1.042 \sim 1.119$ & $<0.001$ \\
(with PNI) & Gender & 2.094 & $0.777 \sim 5.641$ & 0.144 \\
& Coma & 6.203 & $2.376 \sim 16.194$ & $<0.001$ \\
& Focal neurological deficits* & 1.090 & $0.441 \sim 2.690$ & 0.852 \\
& Intracerebral hemorrhage & 3.304 & $1.275 \sim 8.560$ & 0.014 \\
& Straight sinus & 8.994 & $2.267 \sim 35.675$ & 0.002 \\
& PNI & 0.903 & $0.833 \sim 0.978$ & 0.012 \\
\hline
\end{tabular}

CVT cerebral venous thrombosis; $A L C$ absolute lymphocyte count; $A L B$ albumin concentration; $P N I$ prognostic nutritional index; $O R$ odds ratio; $\mathrm{Cl}$ confidence interval

\section{Results}

\section{Study subject characteristics}

Out of 324 patients who were diagnosed with CVST within 30 days of onset to admission and enrolled in the database, 27 patients were excluded: 15 patients younger than 18 years old, 8 patients without complete clinical data and 4 patients lost to follow-up. Therefore, 297 patients were included in the final analysis. The comparison of baseline characteristics and treatment details of included and excluded patients, showed no difference except for age (Table S1).

\section{Characteristics of patients with good and poor prognosis}

During the follow-up period, 33 patients (11.1\%) had poor functional outcomes, including 26 patients who died $(8.8 \%)$. The baseline characteristics of the two groups are shown in Table 1 . The PNI $(49.3 \pm 6.3$ versus $42.3 \pm 6.1, P<0.001$ ) of the poor outcome group considerably decreased. Patients with poor outcomes were older (34.2 \pm 11.6 versus $44.4 \pm 16.3 ; P<0.001)$ compared with the good outcome group. In addition, coma, focal neurological deficits, involvement of straight sinus, and intracerebral hemorrhage in neuroimaging manifestations were more common in patients with adverse prognosis. The

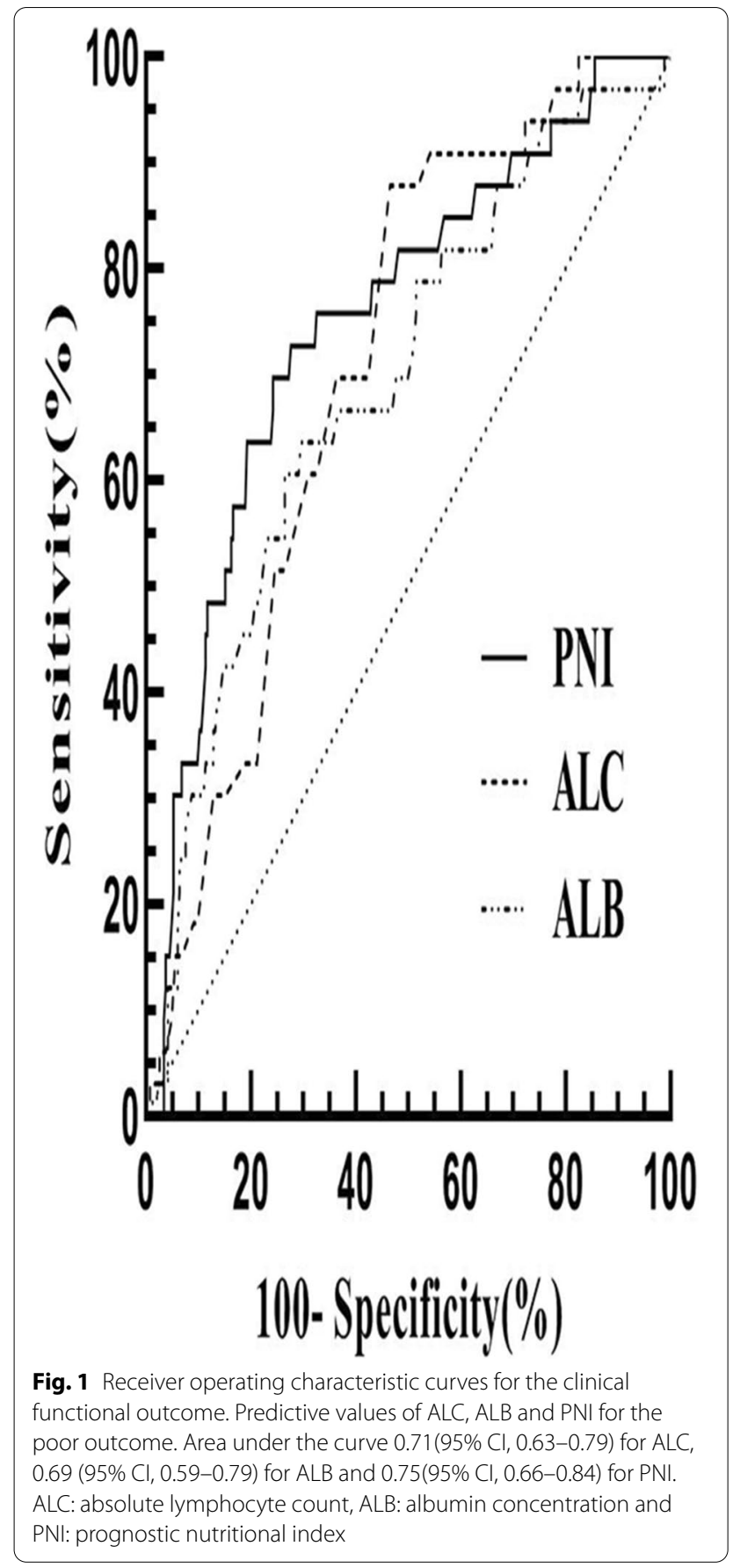

lymphocyte count and albumin concentration in subjects with adverse outcomes were lower than those with good outcomes $(P<0.001)$.

\section{Analysis between PNI and long-term prognosis}

As presented in Table 2, multivariate logistic regression analysis suggested that PNI (odds ratio, 0.903; 95\% CI, 0.833-0.978; $P=0.012$ ) remained significantly 


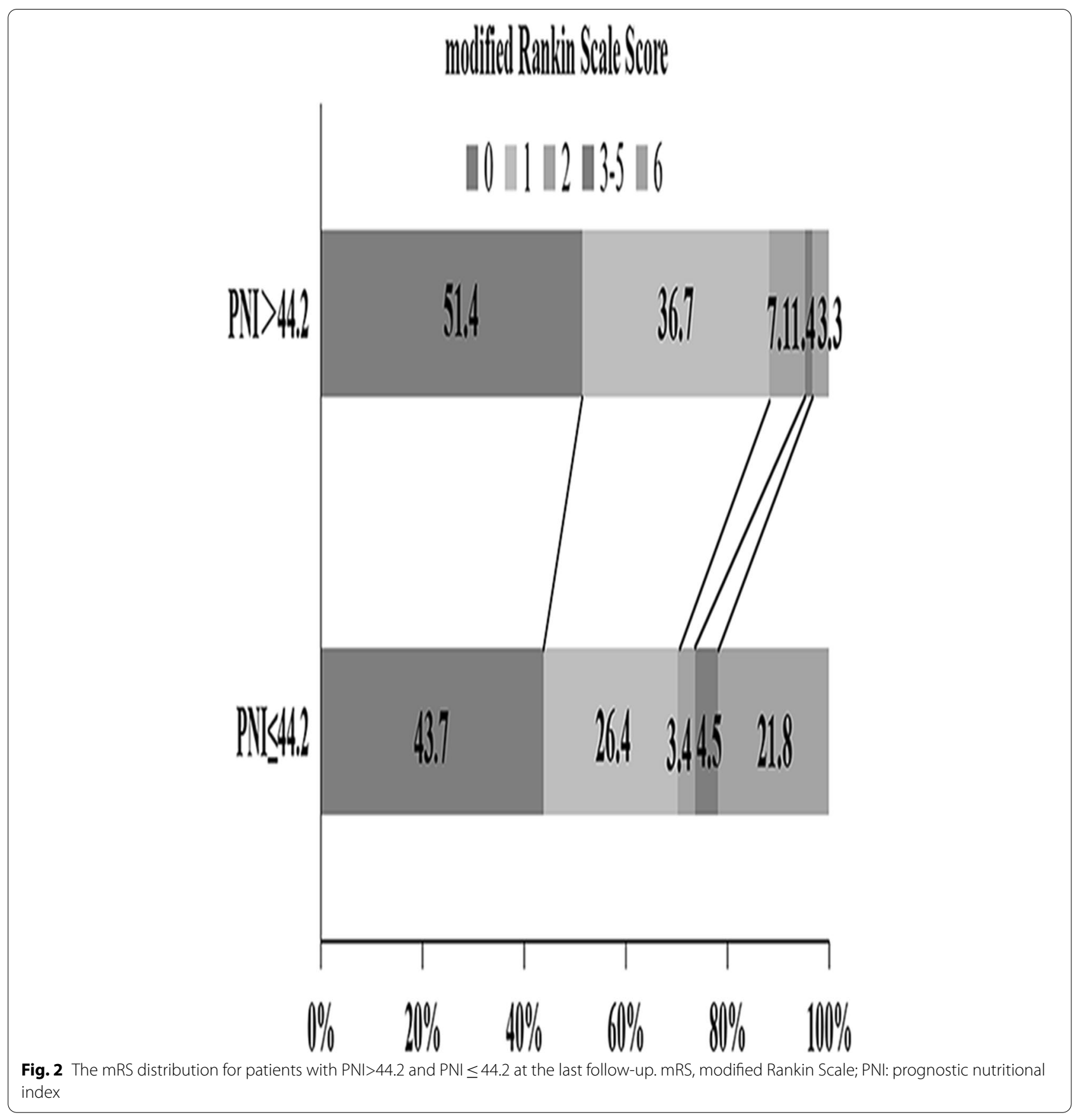

correlated with the functional outcome after the adjustment of potential confounders including age, gender, presence of coma, focal neurological deficits, intracerebral hemorrhage, and straight sinus. According to the ROC curve of laboratory parameters to predict poor results shown in Fig. 1, when 44.2 was set as the cut-off point, PNI showed the best predictive value with the receiver operating characteristic analysis and area under the curve (AUC) of 0.75 (sensitivity: 75.8\% and specificity: 69.7\%), which showed the highest AUC value than those of ALC (AUC 0.71; 95\% CI, 0.63-0.79) and ALB (AUC 0.69; 95\% CI, 0.59-0.79). It indicated that PNI provides a stronger predictive power of poor outcome than its constituent parts. The mRS distribution is shown in Fig. 2. Overall, patients with lower PNI had worse scores on the mRS than higher PNI patients. 

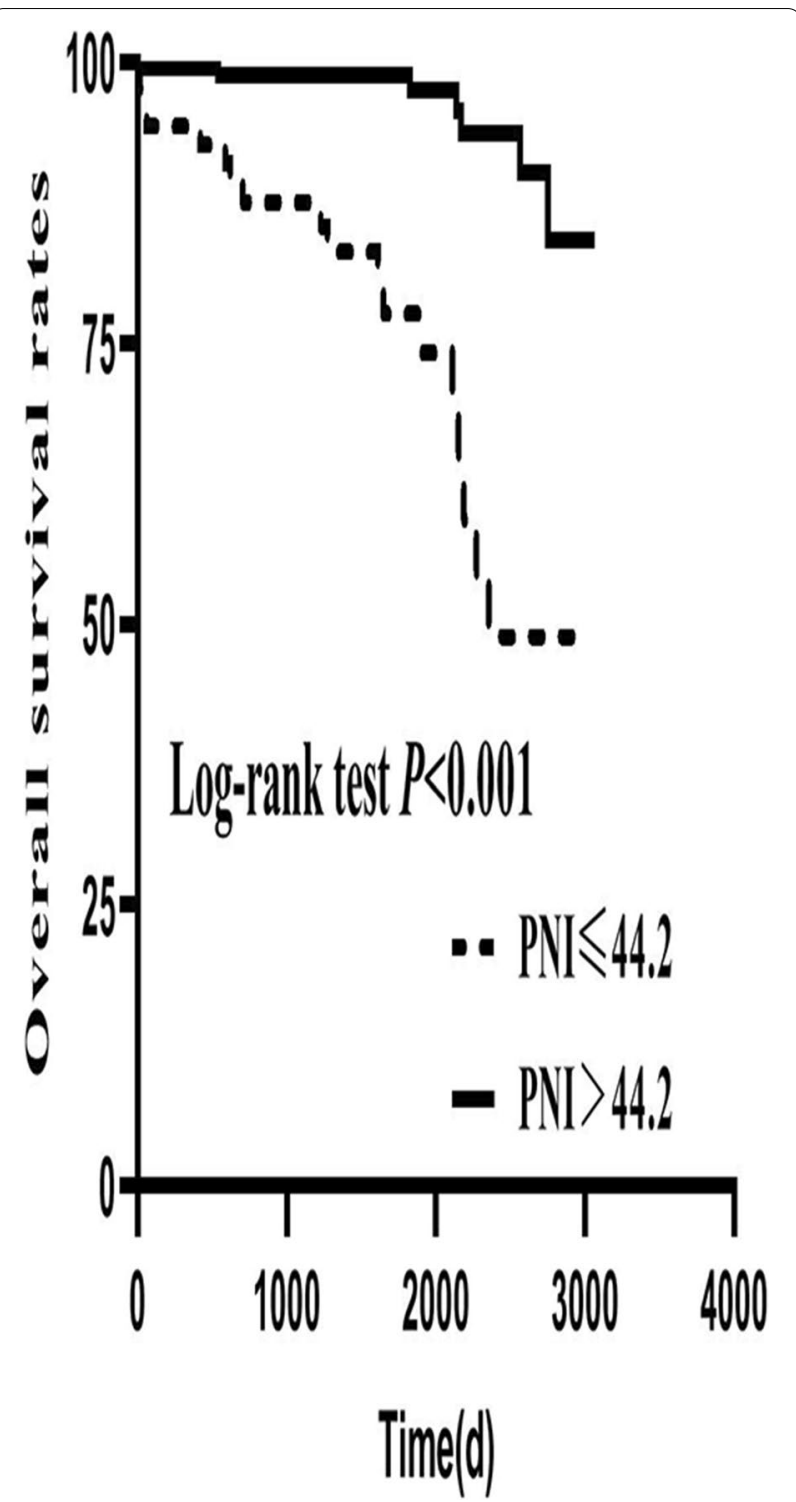

Fig. 3 Kaplan-Meier curves of patients stratified according to the PNI value. The Kaplan-Meier curves showed a significant difference between the PNI $>44.2$ and $\mathrm{PNI} \leq 44.2$ categories (Log-rank test $=32.71, P<0.001)$. PNI: prognostic nutritional index

Kaplan-Meier analysis and log-rank test suggested that the lower the PNI value, the higher the mortality rate $(P<0.001)$ (Fig. 3).

\section{Nomograms to predict the prognosis}

In addition, age, coma, intracranial hemorrhage, straight sinus involvement and PNI were included in the nomogram of the study (Fig. 4) by stepwise logistic regression analysis. The new model showed that older age, coma, intracerebral hemorrhage, straight sinus, and lower PNI were indicators of poor prognosis. The c-index of the model was 0.872 . These findings were similar to those of the previous multivariate logistic model.

\section{Discussion}

In the International Study on Cerebral Vein and Dural Sinus Thrombosis (ISCVT), male gender, age $>37$ years old, mental status disturbance, coma, thrombosis of the deep venous system, intracranial hemorrhage, malignancy, and infection of the central nervous system were independently associated with adverse outcomes at the last follow-up [19]. The VENOST study is the largest national multicenter study. Compared with previous studies, the clinical and imaging results are consistent; however, the susceptibility factors are different. The incidence of puerperium is high, and the use of oral contraceptives is not a common risk factor. The prognosis of malignant tumors, advanced age and hemorrhagic infarction is worse [20]. Our study showed that PNI was a significant and independent predictor of poor prognosis in non-chronic CVST patients.

The current research supports the link between inflammation and the incidence of CVST [21, 22]. A number of studies appeared to reinforce the biological plausibility behind inflammation and the prognosis of cerebral venous thrombosis [23, 24]. The lymphocyte count is an indicator that mediates cellular immunity. It is worth noting that some studies supported the use of lymphocyte count as a prognostic indicator. Lymphocytes are involved in the cellular immunity of various cancers and are related to cancer prognosis [25]. In addition, a previous study has shown that lymphocyte count is a predictive factor of adverse outcomes in patients with AIS [26]. In animal models, immunosuppression induced by stroke could lead to lymphopenia and changes in the ratio of helper T cells $[27,28]$.

Similarly, in human studies, it has also been observed that peripheral blood lymphocytes decreased after stroke, particularly in the acute phase $[29,30]$. Therefore, lower lymphocyte count may be a predictor of adverse outcome. The relationship between lymphocyte count and the outcome of CVST has been confirmed in previous studies, such as platelet to lymphocyte ratio (PLR), lymphocyte to monocyte ratio (LMR) and neutrophil to lymphocyte ratio (NLR) [17, 31, 32].

Hypoalbuminemia is a comprehensive result of inflammation and insufficient intake of protein and calories in patients with chronic diseases. In different clinical settings, hypoalbuminemia has been shown to be a sign of poor prognosis [33-35]. Considering that albumin is a negative acute-- phase protein, its synthesis rate is affected by nutrition and inflammation [36]. More evidence shows that as the severity of inflammation increases, serum albumin levels gradually 


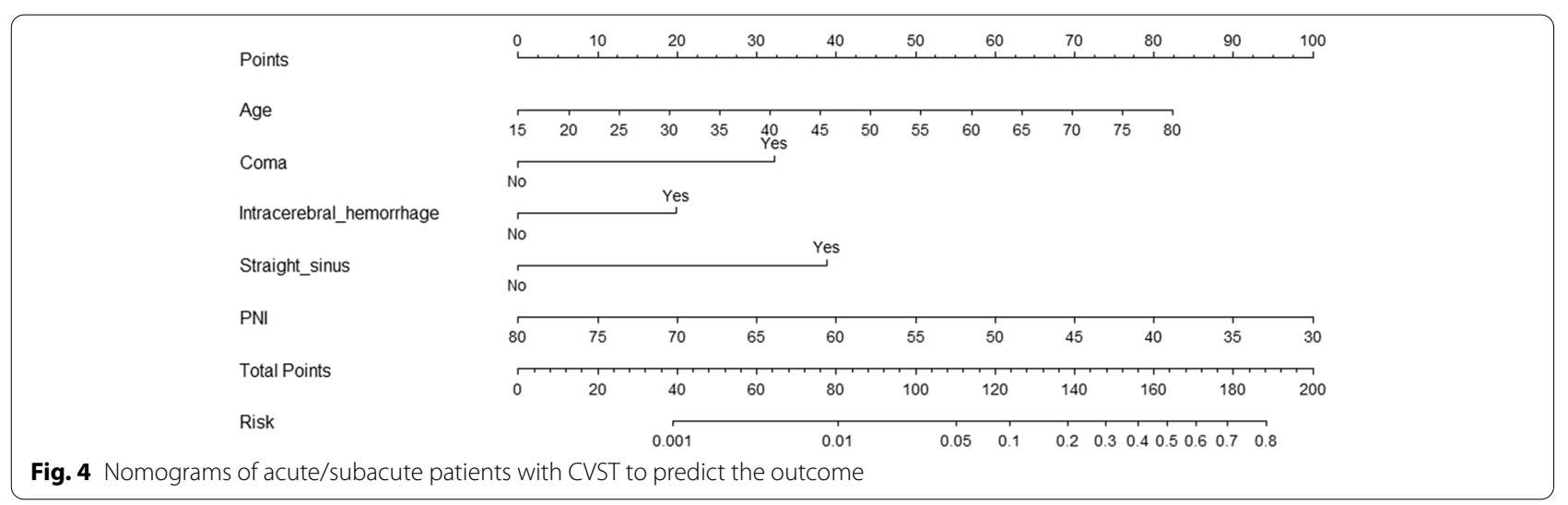

decrease $[37,38]$. Albumin combined with nitric oxide (NO) free radicals has anticoagulant and antithrombotic effects. Due to the increase in the concentration of free lysophospholipids, hypoalbuminemia may affect blood viscosity and the function of endothelial cells [39]. However, serum albumin is not a good indicator of nutrition observation because it is greatly affected by fluid transfer [33]. Many different factors affect serum albumin levels and have shown a lack of sensitive and specific indicators of nutritional status [40].

As for PNI, it combines the lymphocyte count and albumin concentration, reflecting the nutrition, inflammation and immunity status. Therefore, compared with the factors mentioned above, PNI is more stable and representative. PNI was originally reported to be used to evaluate the immune and nutritional status of patients undergoing gastrointestinal tract surgery [18, 41, 42]. Subsequently, PNI has been widely used in prognostic evaluation of a variety of cancers and transplant operations, as well as for patients with various diseases such as myocardial infarction, acute type A aortic dissections and AIS patients receiving intravenous thrombolysis (IVT) [12, 13, 43, 44].

This study further showed that lower PNI increased the risk of poor outcomes in acute/subacute patients with CVST. Additionally, the findings from nomograms by stepwise logistic regression analyses suggested that age, coma, intracerebral hemorrhage, straight sinus involvement, and PNI were also predictors of adverse outcomes in acute/subacute patients with CVST, which further supported the results from multivariate logistic regression analyses. Therefore, the PNI created by combining serum albumin concentration and lymphocyte counts has the ability to assess the nutritional, inflammatory and immune status of patients with acute/subacute CVST. Considering that patients with lower PNI scores in this study have a significantly higher incidence of poor prognosis, it can be considered that appropriate evaluation and implementation of measures to improve nutritional status will help improve the outcome of patients with acute/subacute CVST. Further prospective studies are required to verify this hypothesis.

PNI is easy to obtain because it is calculated using objective laboratory test data. This makes it easy for our achievements to be translated into daily practice. However, several potential limitations should be acknowledged in the present study. Firstly, it is a single center study, and the sample size included in this study is relatively small. This association in the present study also needs to be confirmed and verified in larger multicenter prospective cohort studies. Secondly, compared with arterial stroke, it is difficult to determine the exact onset time in patients with CVST.

\section{Conclusion}

In summary, our study suggested that lower PNI was a potential risk factor in unfavorable functional outcome of patients with acute/subacute CVST.

\section{Abbreviations}

CVST: Cerebral venous sinus thrombosis; PNI: Lower prognostic nutritional index; mRS: Modified Rankin Scale; MI: Myocardial infarction; AIS: Acute ischemic stroke; IVT: Intravenous thrombolysis; CT: Computed tomographic; MRV: Magnetic resonance venography; DSA: Digital subtraction angiography; ALB: Albumin concentration; ALC: Absolute lymphocyte count; ROC: Receiver operating characteristic; AUC: The receiver operating characteristic analysis and area under the curve; ISCVT: International Study on Cerebral Vein and Dural Sinus Thrombosis; PLR: Platelet to lymphocyte ratio; LMR: Lymphocyte to monocyte ratio; NLR: Neutrophil to lymphocyte ratio; NO: Nitric oxide; CVT: Cerebral venous thrombosis; OR: Odds ratio; Cl: Confidence interval.

\section{Supplementary Information}

The online version contains supplementary material available at https://doi. org/10.1186/s12883-021-02436-w.

Additional file 1. 


\section{Acknowledgements \\ None.}

\section{Authors' contributions}

YMX and BS: conception, design and administrative support. JWZ and KL: provision of study materials or patients. SL and HBL: collection and assembly of data. YG, LZ, HF, JW, SLS and YSL: data analysis and interpretation. Manuscript writing: All authors. Final approval of manuscript: All authors.

\section{Funding}

The work was supported by the Grants from the Science and Technology Department of the Henan Province (No. 152102310058 to Dr. Bo Song).

\section{Availability of data and materials}

Study data are available from the corresponding author upon request.

\section{Declarations}

\section{Ethics approval and consent to participate}

The authors are accountable for all aspects of the work in ensuring that questions related to the accuracy or integrity of any part of the work are appropriately investigated and resolved. Informed consent was obtained from all subjects. All methods were carried out in accordance with the relevant guidelines and regulations. The research protocols were approved by the Ethics Committee of the First Affiliated Hospital of Zhengzhou University (Number: 2009-KY-48)

\section{Consent for publication}

Not applicable.

\section{Competing interests}

The authors have no conflicts of interest to declare.

Received: 18 January 2021 Accepted: 5 October 2021

Published online: 21 October 2021

\section{References}

1. Filippidis A, Kapsalaki E, Patramani G. Fountas KN cerebral venous sinus thrombosis: review of the demographics, pathophysiology, current diagnosis, and treatment. Neurosurg Focus. 2009;27(5):E3.

2. Medel R, Monteith SJ, Crowley RW. Dumont AS a review of therapeutic strategies for the management of cerebral venous sinus thrombosis. Neurosurg Focus. 2009;27(5):E6.

3. Chik Y, Gottesman RF, Zeiler SR, Rosenberg J. Llinas RH differentiation of transverse sinus thrombosis from congenitally atretic cerebral transverse sinus with CT. Stroke. 2012:43(7):1968-70.

4. Saposnik G, Barinagarrementeria F, Brown RD Jr, Bushnell CD, Cucchiara B, Cushman M, et al. Diagnosis and management of cerebral venous thrombosis: a statement for healthcare professionals from the American Heart Association/American Stroke Association. Stroke. 2011:42(4):1158-92.

5. Ferro JM, Bousser MG, Canhao P, Coutinho JM, Crassard I, Dentali F, et al. European stroke organization guideline for the diagnosis and treatment of cerebral venous thrombosis - endorsed by the European academy of neurology. European Stroke J. 2017:2(3):195-221.

6. Coutinho JM, Zuurbier SM, Aramideh M. Stam J the incidence of cerebral venous thrombosis: a cross-sectional study. Stroke. 2012:43(12):3375-7.

7. Devasagayam S, Wyatt B, Leyden J. Kleinig T cerebral venous sinus thrombosis incidence is higher than previously thought: a retrospective population-based study. Stroke. 2016;47(9):2180-2.

8. Gulati D, Strbian D. Sundararajan S cerebral venous thrombosis: diagnosis and management. Stroke. 2014;45(2):e16-8.

9. Zhang X, Pang L, Sharma SV, Li R, Nyitray AG, Edwards BJ Malnutrition and overall survival in older patients with cancer. Clin Nutr. 2020

10. Adejumo AC, Adejumo KL, Adegbala OM, Enwerem N, Ofosu A, Akanbi $\mathrm{O}$, et al. Inferior outcomes of patients with acute myocardial infarction and comorbid protein-energy malnutrition. JPEN J Parenter Enteral Nutr. 2020:44(3):454-62.
11. Cai ZM, Wu YZ, Chen HM, Feng RQ, Liao CW, Ye SL, et al. Being at risk of malnutrition predicts poor outcomes at 3 months in acute ischemic stroke patients. Eur J Clin Nutr. 2020;74(5):796-805.

12. Wang SH, Zhai ST. Lin H role of prognostic nutritional index in patients with gastric cancer: a meta-analysis. Minerva Med. 2016;107(5):322-7.

13. Chen QJ, Qu HJ, Li DZ, Li XM, Zhu JJ, Xiang Y, et al. Prognostic nutritional index predicts clinical outcome in patients with acute ST-segment elevation myocardial infarction undergoing primary percutaneous coronary intervention. Sci Rep. 2017:7(1):3285

14. Xiang W, Chen X, Ye W, Li J, Zhang X. Xie D prognostic nutritional index for predicting 3-month outcomes in ischemic stroke patients undergoing thrombolysis. Front Neurol. 2020;11:599.

15. Li S, Liu K, Gao Y, Zhao L, Zhang R, Fang H, et al. Prognostic value of systemic immune-inflammation index in acute/subacute patients with cerebral venous sinus thrombosis. Stroke Vasc Neurol. 2020;5(4):368-73.

16. Meng R, Wang X, Hussain M, Dornbos D 3rd, Meng L, Liu Y, et al. Evaluation of plasma D-dimer plus fibrinogen in predicting acute CVST. International journal of stroke : official journal of the International Stroke Society. 2014;9(2):166-73.

17. Li S, Liu K, Zhang R, Gao Y, Fang H, Liu X, et al. Lower lymphocyte to monocyte ratio is a potential predictor of poor outcome in patients with cerebral venous sinus thrombosis. Stroke Vasc Neurol. 2019;4(3):148-53.

18. Onodera T, Goseki N. Kosaki G [prognostic nutritional index in gastrointestinal surgery of malnourished cancer patients]. Nihon Geka Gakkai Zasshi. 1984:85(9):1001-5.

19. Ferro JM, Canhao P, Stam J, Bousser MG, Barinagarrementeria F. Investigators I prognosis of cerebral vein and dural sinus thrombosis: results of the international study on cerebral vein and Dural sinus thrombosis (ISCVT). Stroke. 2004;35(3):664-70.

20. Duman T, Demirci S, Uluduz D, Kozak HH, Demir S, Misirli CH, et al. Cerebral venous sinus thrombosis as a rare complication of systemic lupus Erythematosus: subgroup analysis of the VENOST study. Journal of Stroke Cerebrovascular Dis. 2019;28(12):104372.

21. Goeijenbier M, van Wissen M, van de Weg C, Jong E, Gerdes VE, Meijers $J C$, et al. Review: viral infections and mechanisms of thrombosis and bleeding. J Med Virol. 2012;84(10):1680-96.

22. Rashad S, Niizuma K, Sato-Maeda M, Fujimura M, Mansour A, Endo H, et al. Early BBB breakdown and subacute inflammasome activation and pyroptosis as a result of cerebral venous thrombosis. Brain Res. 1699:2018:54-68.

23. Wang L, Duan J, Bian T, Meng R, Wu L, Zhang Z, et al. Inflammation is correlated with severity and outcome of cerebral venous thrombosis. J Neuroinflammation. 2018;15(1):329.

24. Aguiar DSD, Pereira-Santos MC, Serra-Caetano A, Lucas NL, Sousa AL, Gabriel D, et al. Blood biomarkers associated with inflammation predict poor prognosis in cerebral venous thrombosis:: a multicenter prospective observational study. Eur J Neurol 2020

25. Kobayashi N, Usui S, Kikuchi S, Goto Y, Sakai M, Onizuka M, et al. Preoperative lymphocyte count is an independent prognostic factor in nodenegative non-small cell lung cancer. Lung Cancer. 2012;75(2):223-7.

26. Xiao J, Qiu QW, Qin C, Tao R, Qiao SY, Chen M, et al. Dynamic changes of peripheral blood lymphocyte subsets in acute ischemic stroke and prognostic value. Brain Behav. 2020:e01919.

27. Prass K, Meisel C, Hoflich C, Braun J, Halle E, WolfT, et al. Stroke-induced immunodeficiency promotes spontaneous bacterial infections and is mediated by sympathetic activation reversal by poststroke T helper cell type 1-like immunostimulation. J Exp Med. 2003;198(5):725-36.

28. Prass K, Braun JS, Dirnagl U, Meisel C. Meisel a stroke propagates bacterial aspiration to pneumonia in a model of cerebral ischemia. Stroke. 2006:37(10):2607-12

29. Haeusler KG, Schmidt WU, Fohring F, Meisel C, Helms T, Jungehulsing GJ, et al. Cellular immunodepression preceding infectious complications after acute ischemic stroke in humans. Cerebrovasc Dis. 2008:25(1-2):50-8.

30. Vogelgesang A, Grunwald U, Langner S, Jack R, Broker BM, Kessler C, et al. Analysis of lymphocyte subsets in patients with stroke and their influence on infection after stroke. Stroke. 2008;39(1):237-41.

31. Akboga YE, Bektas H. Anlar O usefulness of platelet to lymphocyte and neutrophil to lymphocyte ratios in predicting the presence of cerebral venous sinus thrombosis and in-hospital major adverse cerebral events. J Neurol Sci. 2017·380:226-9. 
32. Artoni A, Abbattista M, Bucciarelli P, Gianniello F, Scalambrino E, Pappalardo E, et al. Platelet to lymphocyte ratio and neutrophil to lymphocyte ratio as risk factors for venous thrombosis. Clin Appl Thromb Hemost. 2018;24(5):808-14.

33. Franch-Arcas $\mathrm{G}$. The meaning of hypoalbuminaemia in clinical practice. Clin Nutr. 2001;20(3):265-9.

34. Oduncu V, Erkol A, Karabay CY, Kurt M, Akgun T, Bulut M, et al. The prognostic value of serum albumin levels on admission in patients with acute ST-segment elevation myocardial infarction undergoing a primary percutaneous coronary intervention. Coron Artery Dis. 2013;24(2):88-94.

35. Bielewicz J, Kurzepa J, Czekajska-Chehab E, Kamieniak P, Daniluk B, Bartosik-Psujek $\mathrm{H}$, et al. Worse neurological state during acute ischemic stroke is associated with a decrease in serum albumin levels. J Mol Neurosci. 2016;58(4):493-6.

36. Kirsch $R$, Frith $L$, Black E. Hoffenberg R regulation of albumin synthesis and catabolism by alteration of dietary protein. Nature. 1968;217(5128):578-9.

37. Ishida S, Hashimoto I, Seike T, Abe Y, Nakaya Y. Nakanishi H serum albumin levels correlate with inflammation rather than nutrition supply in burns patients: a retrospective study. J Med Investig. 2014;61(3-4):361-8.

38. Eckart A, Struja T, Kutz A, Baumgartner A, Baumgartner T, Zurfluh S, et al. Relationship of nutritional status, inflammation, and serum albumin levels during acute illness: a prospective study. Am J Med. 2020;133(6):713-22 e717.

39. Joles JA, Willekes-Koolschijn N. Koomans HA Hypoalbuminemia causes high blood viscosity by increasing red cell lysophosphatidylcholine. Kidney Int. 1997;52(3):761-70.
40. Levitt DG. Levitt MD human serum albumin homeostasis: a new look at the roles of synthesis, catabolism, renal and gastrointestinal excretion, and the clinical value of serum albumin measurements. Int J Gen Med. 2016;9:229-55.

41. Nozoe T, Ninomiya M, Maeda T, Matsukuma A, Nakashima H. Ezaki T prognostic nutritional index: a tool to predict the biological aggressiveness of gastric carcinoma. Surg Today. 2010;40(5):440-3.

42. Buzby GP, Mullen JL, Matthews DC, Hobbs CL. Rosato EF prognostic nutritional index in gastrointestinal surgery. Am J Surg. 1980;139(1):160-7.

43. Kim CY, Kim SY, Song JH, Kim YS, Jeong SJ, Lee JG, et al. Usefulness of the preoperative prognostic nutritional index score as a predictor of the outcomes of lung transplantation: a single-institution experience. Clin Nutr. 2019;38(5):2423-9.

44. Lin Y, Chen Q, Peng Y, Chen Y, Huang X, Lin L, et al. Prognostic nutritional index predicts in-hospital mortality in patients with acute type a aortic dissection. Heart Lung. 2021;50(1):159-64.

\section{Publisher's Note}

Springer Nature remains neutral with regard to jurisdictional claims in published maps and institutional affiliations.
Ready to submit your research? Choose BMC and benefit from:

- fast, convenient online submission

- thorough peer review by experienced researchers in your field

- rapid publication on acceptance

- support for research data, including large and complex data types

- gold Open Access which fosters wider collaboration and increased citations

- maximum visibility for your research: over $100 \mathrm{M}$ website views per year

At BMC, research is always in progress.

Learn more biomedcentral.com/submissions 